\title{
Evaluation of Polymethylene Waxes as Conditioning Agents in Hair Relaxers
}

\author{
R. B. Shalvoy, S. Vermeulen, ** and S. Wilford*** \\ * Arch Chemicals, 350 Knotter Drive, Cheshire, CT 06410 \\ ** Arch Personal Care Products, 184 Edison Crescent, Hennopspark X7, Centurion, Gauteng, South \\ Africa \\ *** Arch Personal Care Products, 70 Tyler Place, South Plainfield, NJ 07080
}

The process of straightening (relaxing) naturally curly hair requires the use of highly alkaline ingredients $(\mathrm{NaOH})$ in the relaxer system. Such treatments leave the hair in a damaged condition with a dull, dry appearance. Conditioners in the relaxer treatment restore good appearance and feel to the hair. The use of polymethylene (Fischer - Tropsch) waxes has been suggested as an effective and stable alternative to quaternized ammonium compounds as the conditioning agent [1]. This study compares the effects of various conditioner ingredients on the appearance of relaxed hair. The restoration of a smooth, undamaged cuticle structure to the surface of the hair is evaluated by SEM imaging using both normal and a high angle of incidence of the electron beam to the hair fiber axis. Observations of shine, feel and smoothness can be understood in light of the damage or lack thereof to the cuticle structure.

Ethnic (African) hair was relaxed using ten different formulations some of which used polymethylene wax as a conditioner. Relaxers using polymethylene wax gave excellent conditioning providing a smooth feel and shiny appearance. The commercial relaxers generally gave good results although there was some variation in the results observed. The relaxed hair was then examined by SEM to understand the causes for these results and to more fully examine the effects of polymethylene wax on the final hair surface structure.

$1 \mathrm{~cm}$ long pieces were cut from the middle of four hair fibers selected randomly from the treated swatches. The samples were first examined in a conventional manner with the electron beam normal to the hair fiber axis [2]. This approach provided a good overall view of the cuticle structure, but did not show details of the cuticle structure well. After this initial observation, the hair samples were tilted to provide a $75^{\circ}$ electron beam angle of incidence to the hair fiber looking from the distal towards the proximal end of the hair fiber. This view provided a closer look at the cuticle structure which allowed better evaluation of cuticle lifting and the deposition of the conditioning agents.

Untreated (tightly curled) ethnic hair is shown in Fig. 1. A moderate degree of surface roughness and irregularities can be seen in both views (normal and angled). The angled position however gives a better view of the cuticle structure with cuticle overlapping and lifting clearly seen. The roughness will cause reduced shine, difficulty in combing and a rough overall feel. Hair that had been treated with the polymethylene wax conditioner (Fig. 2) has a smooth coated appearance with a minimum of damage and lifting of cuticles. This hair had the best perceived conditioning. Fig. 3 shows two angled views of hair treated with commercial products, one with an excellent surface structure and one showing areas with irregularities that diminished the perceived conditioned effect.

References

[1] S. Vermeulen et al., J. Cosmet. Sci. 55 (Suppl.) (2004) S181. 
[2] C. R. Robbins, Chemical and Physical Behavior of Human Hair, $4^{\text {th }}$ Ed., Springer Verlag, Frankfurt, Germany, 2001.

[3] The authors wish to thank J. V. Gruber (Arch Chem., S. Plainfield) for helpful discussions.
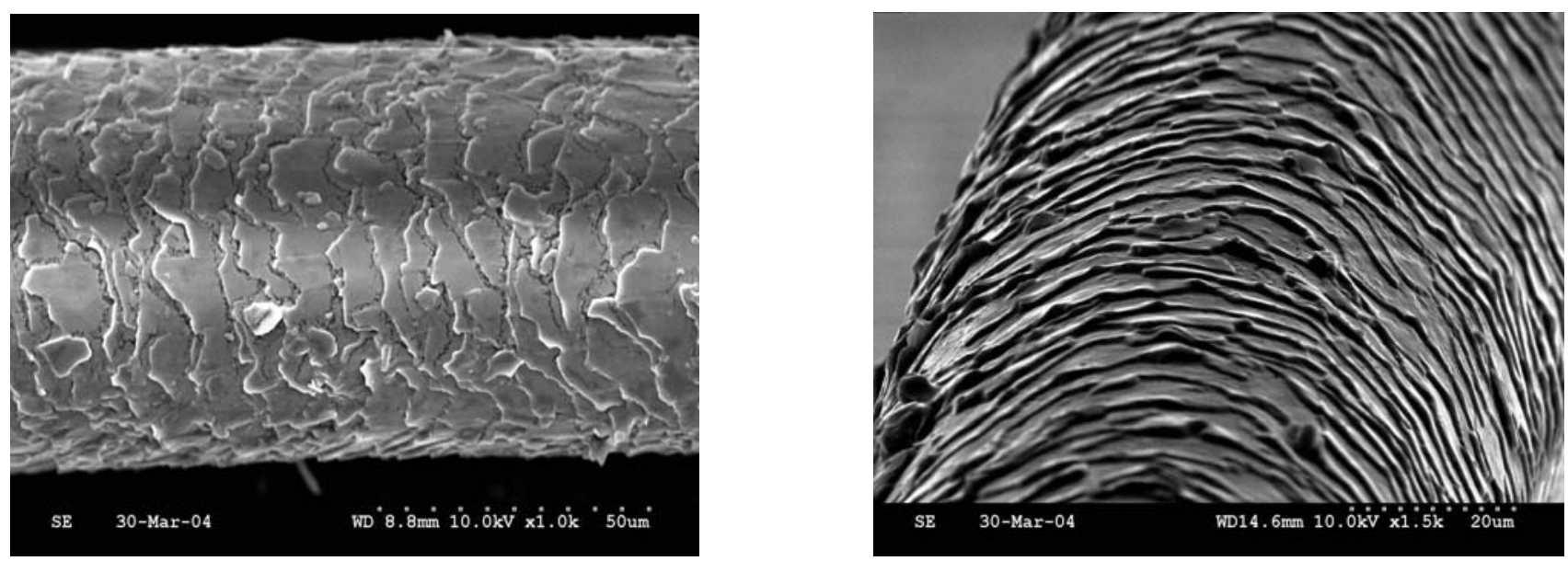

FIG. 1. Untreated Ethnic (African) Hair. Normal and $75^{\circ}$ beam incidence views shown.
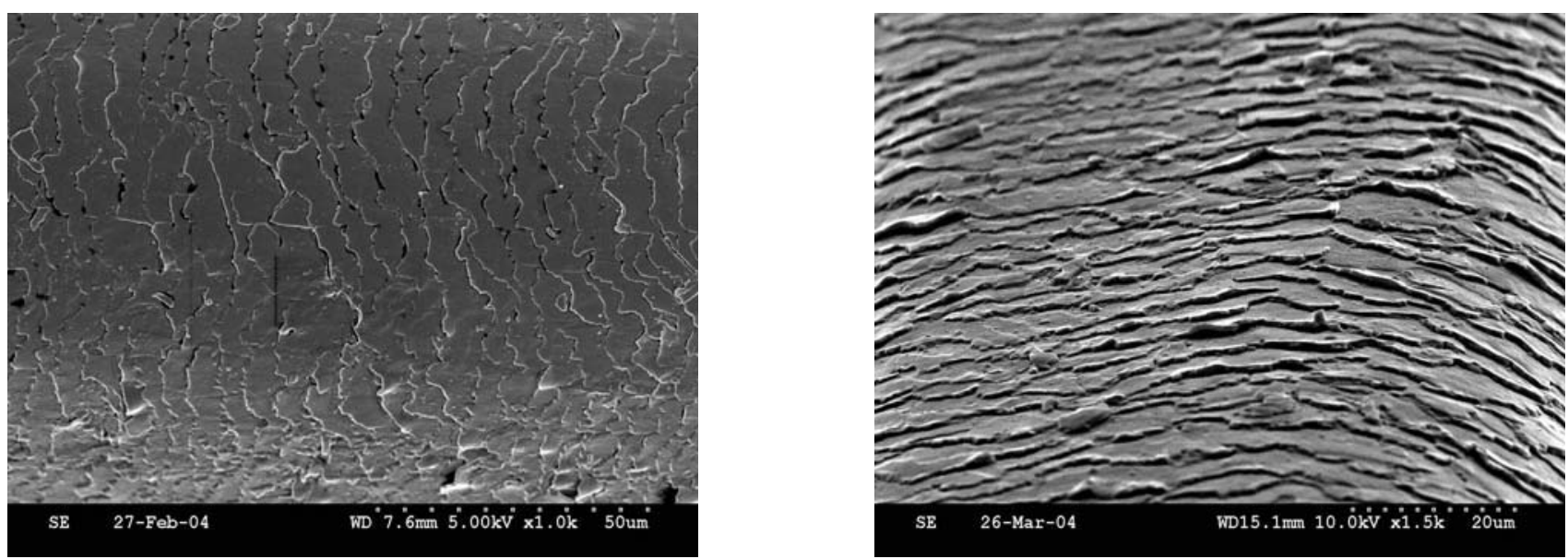

FIG. 2. Hair Treated with Polymethylene Wax Conditioner. Normal and $75^{\circ}$ incidence views.
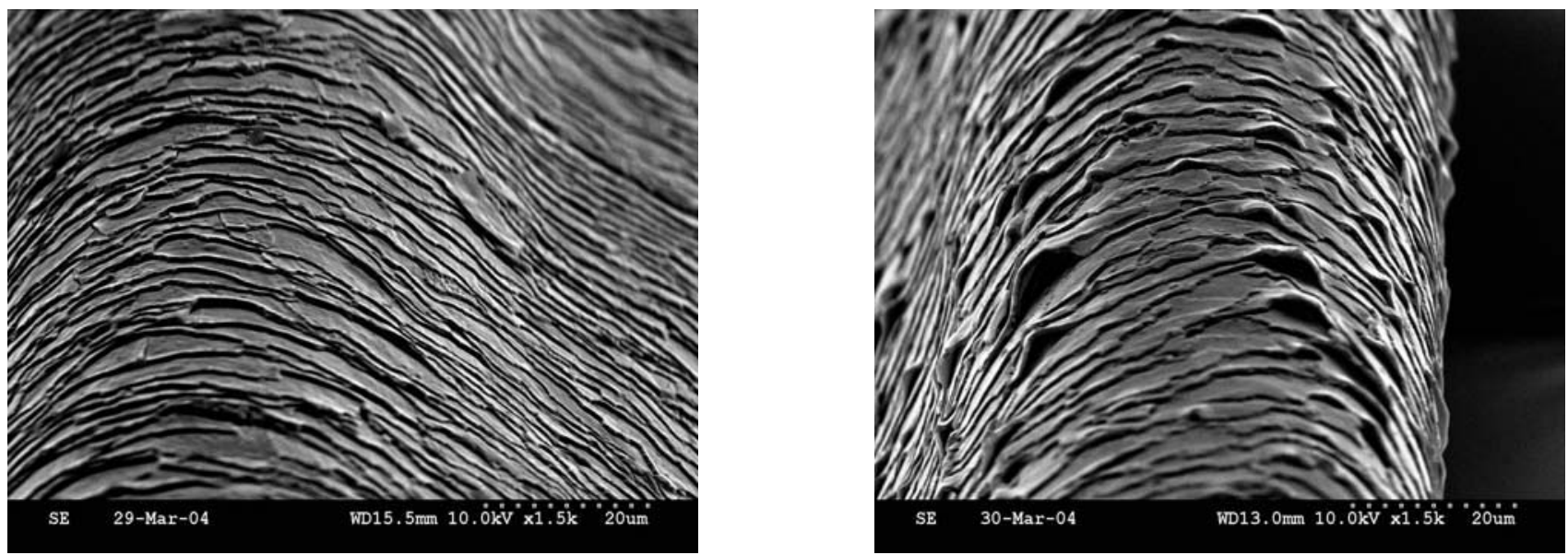

FIG. 3. Hair Treated with Commercial Relaxers (non-wax) $75^{\circ}$ incidence views. 